\title{
MODELLING OF BIOMETRIC IDENTIFICATION SYSTEM WITH GIVEN PARAMETERS USING COLORED PETRI NETS
}

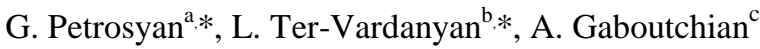 \\ ${ }^{a}$ Institute for Informatics and Automation Problems of NAS RA, Yerevan, Armenia, \\ petrosyan_gohar@list.ru \\ ${ }^{\mathrm{b}}$ International Scientific - Educational Centre of NAS RA, Yerevan, Armenia, \\ lilit@sci.am \\ ${ }^{\mathrm{c}}$ Moscow State Medical-Stomatological University, 127473 Moscow, Russia - \\ armengaboutchian@mail.ru
}

\author{
Commission II, WG II/10
}

KEY WORDS: Photogrammetry, Colored Petri Nets, Automation, Feature Detection, Biometry, Odontology, Tooth Structure.

\begin{abstract}
:
Biometric identification systems use given parameters and function on the basis of Colored Petri Nets as a modelling language developed for systems in which communication, synchronization and distributed resources play an important role. Colored Petri Nets combine the strengths of Classical Petri Nets with the power of a high-level programming language. Coloured Petri Nets have both, formal intuitive and graphical presentations. Graphical CPN model consists of a set of interacting modules which include a network of places, transitions and arcs. Mathematical representation has a well-defined syntax and semantics, as well as defines system behavioural properties. One of the best known features used in biometric is the human finger print pattern. During the last decade other human features have become of interest, such as iris-based or face recognition. The objective of this paper is to introduce the fundamental concepts of Petri Nets in relation to tooth shape analysis. Biometric identification systems functioning has two phases: data enrollment phase and identification phase. During the data enrollment phase images of teeth are added to database. This record contains enrollment data as a noisy version of the biometrical data corresponding to the individual. During the identification phase an unknown individual is observed again and is compared to the enrollment data in the database and then system estimates the individual. The purpose of modeling biometric identification system by means of Petri Nets is to reveal the following aspects of the functioning model: the efficiency of the model, behavior of the model, mistakes and accidents in the model, feasibility of the model simplification or substitution of its separate components for more effective components without interfering system functioning.

The results of biometric identification system modeling and evaluating are presented and discussed.
\end{abstract}

\section{INTRODUCTION}

The development of high-speed computers has greatly increased the use and usefulness of modeling. By representing a system as a mathematical model, converting that model into instructions for a computer, and running the computer, it is possible to model larger and more complex systems than ever before. This has resulted in considerable study of computer modeling techniques and of computers themselves. Computers are involved in modeling in two ways: as a computational tool for modeling and as a subject of modeling. In modern society informational communication and confidential usage of it, have become a common matter and is quite used by many. One of the important areas of application are biometry and Petri net theory. Biometric technologies are predominantly used to authenticate individuals in applications such as access control - to enter a facility or sign-on to a personal computer, identity management - search photographs for multiple aliases (Visa applications), or time and attendance. Biometric is defined as the automated recognition of behavioural and physiological characteristics of an individual. The core tasks of biometric security are person's reliable identification and authentication according to his biometric data. The main problem of Petri net is modeling of real-time systems by optimization of the processes with clear description.

* Corresponding author
Petri nets are a tool for the study of systems. Petri net theory allows a system to be modeled by a Petri net, a mathematical representation of the system. Analysis of the Petri net can then, hopefully, reveal important information about the structure and dynamic behaviour of the modeled system. This information can then be used to evaluate the modeled system and suggest improvements or changes. Thus, the development of a theory of Petri nets is based on the application of Petri nets in the modeling and design of systems.

Therefore, Petri Net computer system gives opportunities to study properties, use them to solve practical problems, mainly those issues that are related to information processing models, paralleled with sources and consider to be an important issue (Peterson, 1981; Jensen, 1996). Till now, it has been shown that the task has fundamental interpretation of information theory (Ahlswede, 1993; Pankanti, 2002).

\section{A BRIEF DESCRIPTION OF PETRI NETS}

The birth of Petri Nets was Petri's dissertation, but most work in the United States is also based on the final report of the Information System Theory Project which translated Petri's dissertation into English as well as extending the work considerably. The "Events and Conditions" paper by Holt and Commoner is also an important part of the early works. Petri presented a short paper to the 1962 IFIP Congress which was 
printed in the proceedings. This paper is based on the ideas in his dissertation.

Petri Net consists of three types of components: places (circles), transitions (rectangles) and arcs (arrows):

- $\quad$ Places represent possible states of the system;

- Transitions are events or actions which cause the change of state;

- Every arc simply connects a place with a transition or a transition with a place.

A Change of State is denoted by a movement of token(s) (black dots) from place(s) to place(s); and is caused by the firing of a transition. The firing represents an occurrence of the event or an action taken. The firing is subject to the input conditions, denoted by token availability. A transition is fireable or enabled when there are sufficient tokens in its input places. After firing, tokens will be transferred from the input places (old state) to the output places, denoting the new state.

Event is an action which occurs in the system. The appearance of the events governs the state of the system. The state of the system can be described by multiple conditions.

The condition is a predicate or a logical description of the states of the system. The condition can admit true or false value. As the events are actions they can take place. In order the event occurs, is necessary appropriate existence of the conditions. They call these conditions the preconditions of the event. The appearance of the event can bring the break of the preconditions and bring to other carrying of the condition-postconditions.

\subsection{Definition}

Petri Net $M(C, \mu)$ pair, where $C=(P, T, I, O)$ is the network structure and $\mu$ is the network condition.

In structure $C$ of a $P$-positions, $T$-transitions are finite sets. $I: T \rightarrow P^{\infty}, O: T \rightarrow P^{\infty}$ are the input and output functions, respectively, where $P^{\infty}$ are all possible collections (repetitive elements) of $P . \mu: P \rightarrow N_{0}$ is the function of condition, where $N_{0}=\{0,1, \ldots\}$ is the set of integers.

As an example of Petri Net let's consider the simple problem of modeling of a Producer-Consumer System.

A producer-consumer system, consist of one producer, two consumers and one storage buffer with the following conditions:

- The storage buffer may contain at most 5 items;

- The producer sends 3 items in each production;

- At most one consumer is able to access the storage buffer at one time;

- Each consumer removes two items when accessing the storage buffer.

The Petri Net is showed in the Figure 1, which simulates the model of Producer-Consumer. In this Petri net, every place has a capacity and every arc has a weight. This allows multiple tokens to reside in a place to model more complex behavior.

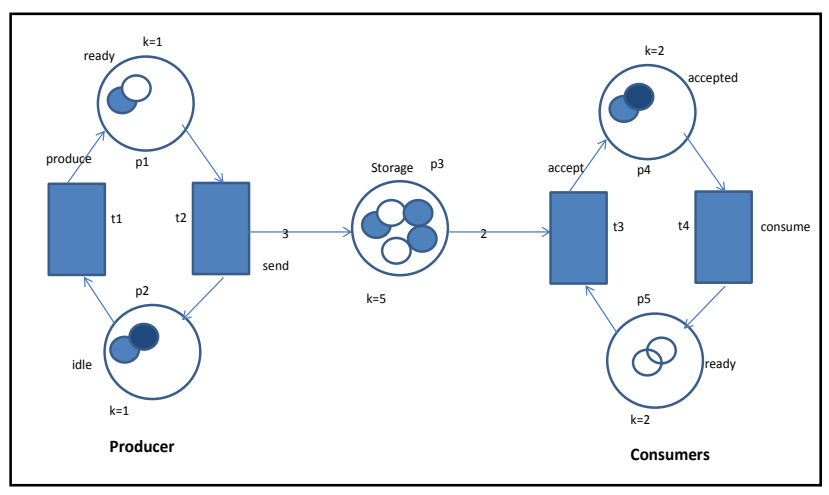

Figure 1. Producer-consumer system scheme.

The usage of places and transitions depends on the system being modelled. Places could represent resource status or operations. Arcs often represent the flow of data or resources. Transitions could represent the start/finish of processes. In terms of simulations, transitions can be used to model both activities and events. Activities can be thought of as the processes and logic of the system, while events occur at a single point in time and cause a change in the state of a system. In fact, a transition may act as a super-process consisting of many sub-processes. In this way hierarchical nets come into play.

\section{A BRIEF DESCRIPTION OF COLORED PETRI NET}

Colored Petri Net (CPN) is a Classical Petri Net modern expansion which was created by K. Jensen. Colored Petri Net (CP-nets or CPNs) is a modelling language developed for systems in which communication, synchronization and resource sharing play an important role. CP-nets combine the strengths of Classical Petri Nets with the strengths of a high-level programming language (Jensen, 1992; Ullman, 1998).

The vast majority of IT systems today can be characterized as concurrent and distributed in that their operation inherently relies on communication, synchronization, and resource sharing between concurrently executing software components and applications. This development was accelerated first through the pervasive presence of the Internet as a communication infrastructure and more recently by cloud- and Web-based services, mobile applications, and multicore computing architectures.

Colored Petri Net is a graphical oriented language for design, specification, simulation and verification of systems (Jensen, 1992; Jensen, 1996; Ullman, 1998). It is in particular wellsuited for systems that consist of a number of processes which communicate and synchronize. Typical examples of application areas are communication protocols, distributed systems, automated production systems, work flow analysis and VLSI chips (very large scale integration, from $10^{6}$ to $10^{7}$ transistors).

Formal definition for Colored Petri Net is presented in the literature (Jensen, 1992; Jensen, 1996; Petrosyan et al., 2015). In the classical or traditional Petri Net tokens do not differ from each other, we can say that they are colourless. Unlike Classical Petri Net, in Colored Petri Net a position can contain tokens of arbitrary complexity - note, lists, etc., that makes reliable models more possible. The Design/CPN system is used for modelling "NOKIA" phones, in order to find out the unwanted functional interactions (Jensen, 1992; Jensen, 1996). 
If briefly describe the scope of the Colored Petri Nets, we can say that: CPNs have a graphical representation, are very general and can be used to describe a large variety of different systems, CPNs have an explicit description of both states and actions, offer hierarchical descriptions, interactive simulations where the results are presented directly on the CPN diagram, CPNs have computer tools supporting their drawing, simulation and formal analysis.

\subsection{Definition}

A Colored Petri Net is a tuple $C P N=(\Sigma, P, T, A, N, C, G, E, I)$ satisfying the following requirements:

(i) $\quad \Sigma$ is a finite set of non-empty types, called colorsets.

(ii) $P$ is a finite set of places.

(iii) $T$ is a finite set of transitions.

(iv) $A$ is a finite set of arcs such that:

- $P \cap T=P \cap A=T \cap A=\emptyset$

(v) $N$ is a node function. It is defined from $A$ into $P \times T \cup T \times P$.

(vi) $C$ is a color function. It is defined from $P$ into $\Sigma$.

(vii) $G$ is a guard function. It is defined from $T$ into expressions such that:

- $\forall t \in T:[\operatorname{Type}(G(t))=B$ ool $\wedge$ Type $(\operatorname{Var}(G(t))) \subseteq \Sigma]$

(viii) $E$ is an arc expression function. It is defined from $A$,by expressions such that:

- $\forall a \in A:\left[\right.$ Type $(E(a))=C(p(a)){ }_{M S} \wedge$ Type $\left.(\operatorname{Var}(E(a))) \subseteq \Sigma\right]$ where $\mathrm{p}(\mathrm{a})$ is the place of $N(a)$

(ix) $\mathrm{I}$ is an initialization function. It is defined from $\mathrm{P}$ into closed expressions such as:

- $\forall p \in P:\left[\operatorname{Type}(I(p))=C(p){ }_{M S}\right]$.

Colored Petri Net consists of the following components:

- The ellipses and the circles are called places. They describe the states of the system (buffers).

- The rectangles are called transitions. They describe the actions (processes).

- The arrows are called arcs. The arc expressions describe how the state of the CPN changes when the transitions occur.

- $\quad$ Each place contains a set of markers called tokens, each of these tokens carries a data value, which belongs to a given type.

Consider the simple example of a Colored Petri Net, which is shown in Figure 2. P1 and P3 positions include tokens of $\mathrm{R}$ type in Fig.2, and P2, P4 positions include tokens of B type. After $\mathrm{T} 1$ transition firing transfers a token of $\mathrm{R}$ type by $(\mathrm{P} 1, \mathrm{~T} 1)$ arc, and transfers a token of B type by (P2, T1) arc (that is, unlike Classical Petri Nets the tokens aren't homogeneous). Two tokens of R type are transfer by (T1, P3) arc, and there appear two tokens in P3. Before T1 transition firing, the state of the system is described by vector of state $S 1=(1,2,0,1)$, and after $\mathrm{T} 1$ transition firing will be $\mathrm{S} 2=(0,1,2,1)$. Therefore, in the result of permissible transitions of firing we will have the set of reachable states of the system.

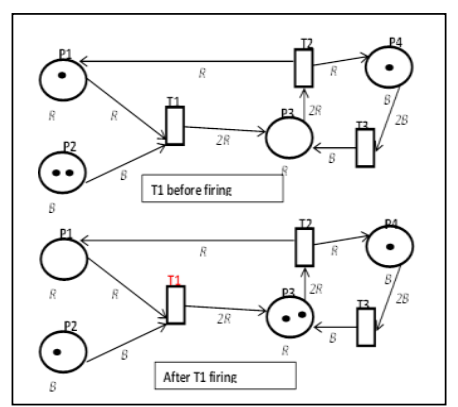

Figure 2. The simple example of a Colored Petri Net

\section{THE MODEL OF BIOMETRIC IDENTIFICATION SYSTEM}

Biometry, being, as a scientific discipline, a part of biology and applied statistics, applies methods of mathematical statistics to biological data analysis and research planning. Francis Galton and Karl Pearson in late XIX, early XX centuries have laid the fundamentals of the discipline which was subsequently developed by their followers. A large number of their scientific works dealt with biology, which has provided for application of biometric in anthropology (Zubov, 2006; Hilson, 2003).

Biometry is often used by different companies, governmental systems, military forces, border control, hospitals, banks etc. to either verify a person's identity, for physical access control, computer log-in, welfare disbursement, international border crossing and national ID cards, e-passports, allowing access to certain building area or to identify individuals to retain information about them, i.e. criminals, forensics, etc. In automobiles, biometric is being installed to replace keys for keyless entry and keyless ignition.

Biometry has been widely applied to anthropological studies. Today identification of teeth is found to be relevant to different applications, among such - anthropological ethnic studies. In the article the project of biometric identification system is proposed, which detection features are based on given parameters analysis. The system based on Petri Nets which are fitted to describe actions and conditions of the system.

The objective of a biometrical identification system is to identify individuals on the basis of physical (passive or active) features. One of the oldest and probably best known of such features is the human fingerprint. One can safely say that for a long time fingerprinting-based identification and biometrical identification have been seen as one and the same thing. In this article for biometric value we take human teeth.

Biometric identification systems were studied by O'Sullivan and Schmid (O'Sullivan and Schmid., 2004; WIllems et al., 2003). They assumed storage of biometric enrollment sequences in the clear and determined the corresponding identification capacity. Later Trucel (Trucel, 2006) analyzed the trade-off between the capacity of a biometric identification system and the storage space (compression rate) required for the biometric templates. It should be noted that Trucel's method realizes a kind of privacy protection scheme. Recall that secrecy capacity introduced by Ahlswede and Csiszar (Ahlswede and Csiszar, 1993) can be regarded as the amount of common secret 
information that can be obtained in an authentication system in which helper data are (publicly) available. Interestingly this secrecy capacity, which is equal to the mutual information between enrollment and authentication biometric sequences in the biometric setting, equals the identification capacity found by O'Sullivan, Schmid and Willems et al.

Depending on given parameters and features teeth identification system is able to classify images for different application, among such biometric, dental or anthropological can be presented. Coloured Petri Nets are best suited to analyze system functioning, error eliminating, validation and verification of biometric data. In our research we use digital images of separate teeth obtained by means of photogrammetric methods, images of teeth obtained by dental arch 3D model segmentation (Figure 3) and images of teeth obtained from segmented dental arch plaster models (Figure 4).

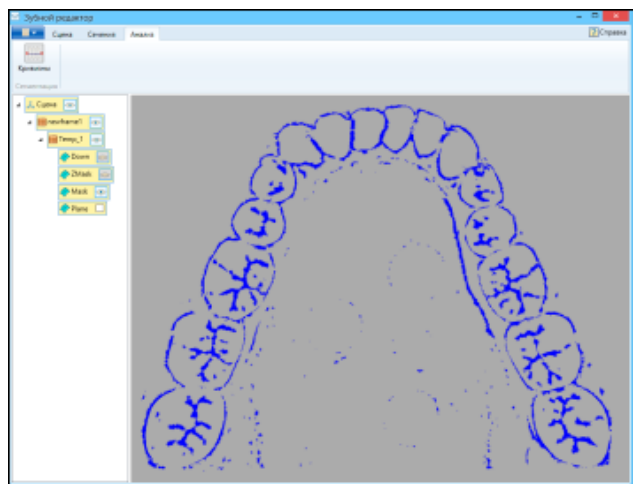

Figure 3. A segmented image of lower dental arch.

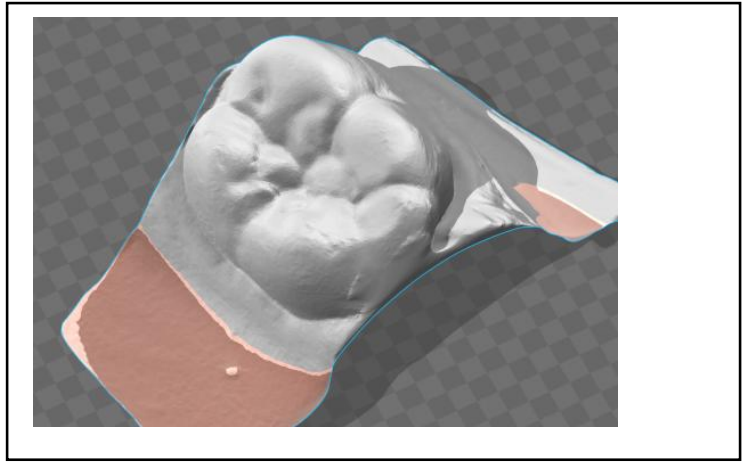

Figure 4. An image of a lower molar - scan of a segmented stone cast model.

\subsection{Notations and Definitions}

Let $X, Y, Z, S$ be finite sets and $W$ is a family of discrete memoryless channels $W_{s}: X \rightarrow Y$, with input alphabet $X$ and output alphabet. The $s$ is the channel state, varying in dependently in each moment of the channel action with the same known $\mathrm{PD} Q(s)$ on $S$.

There are $F$ teeth and each tooth has an index $f=\{1,2, \ldots, F\}$.
A biometric data sequence $x(f)=\left\{x_{1}, x_{2}, \ldots, x_{n}\right\}$, where $x \in X, n=\overline{1, \ldots, N}$ corresponds to each teeth $f$. All these sequences are supposed to be generated at random with a given probability distribution

$$
P^{N}(x)=\prod_{n=1}^{N} P\left(x_{n}\right), x \in X^{N}
$$

\subsection{Enrollment phase}

Let we have the stationary and discrete channel which does not have memory $W(y \mid x, s)$ with given parameter. In this phase all biometric data sequences $x(f)$ are observed via this channel. The state of the channel is changed by following probability distribution $Q^{N}(s)$ it is mean

$$
\begin{gathered}
W^{N}(y \mid x, s)=\prod_{n=1}^{N} W\left(y_{n} \mid x_{n}, s_{n}\right) \\
Q^{N}(s)=\prod_{n=1}^{N} Q\left(s_{n}\right), x \in X^{N}, y \in Y^{N}, s \in S^{N}
\end{gathered}
$$

The resulting $y(f)$ enrollment output sequences for all $f=\{1,2, \ldots, F\}$ are stored in a database (Fig.5).

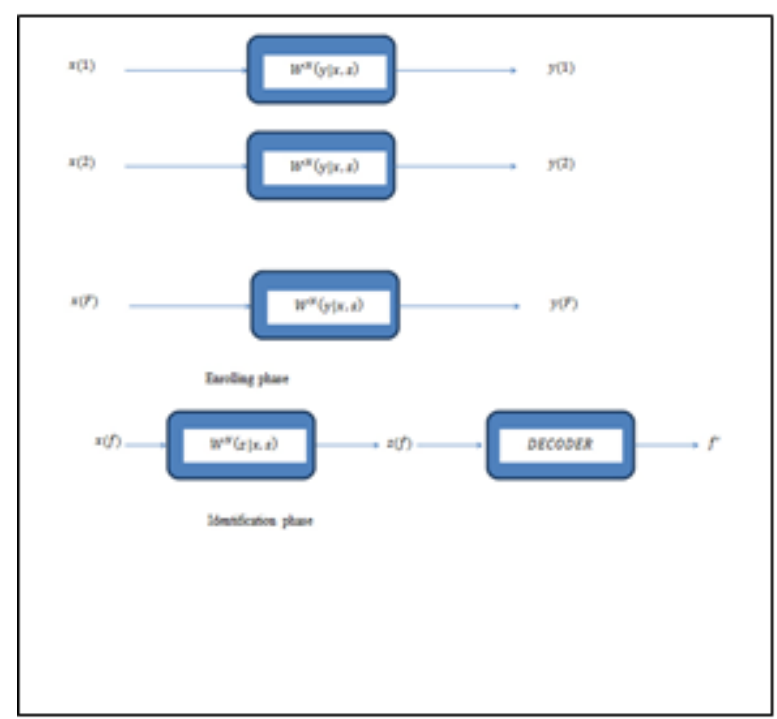

Figure 5. The Model of Biometric Identification System with Given Parameters

\subsection{Identification phase}

In the identification phase the biometric data sequence of an unknown teeth observed via a same memoryless channel $W(z \mid x, s)$ with given parameter.

$$
W^{N}(z \mid x, s)=\prod_{n=1}^{N} W\left(y_{n} \mid x_{n}, s_{n}\right)
$$




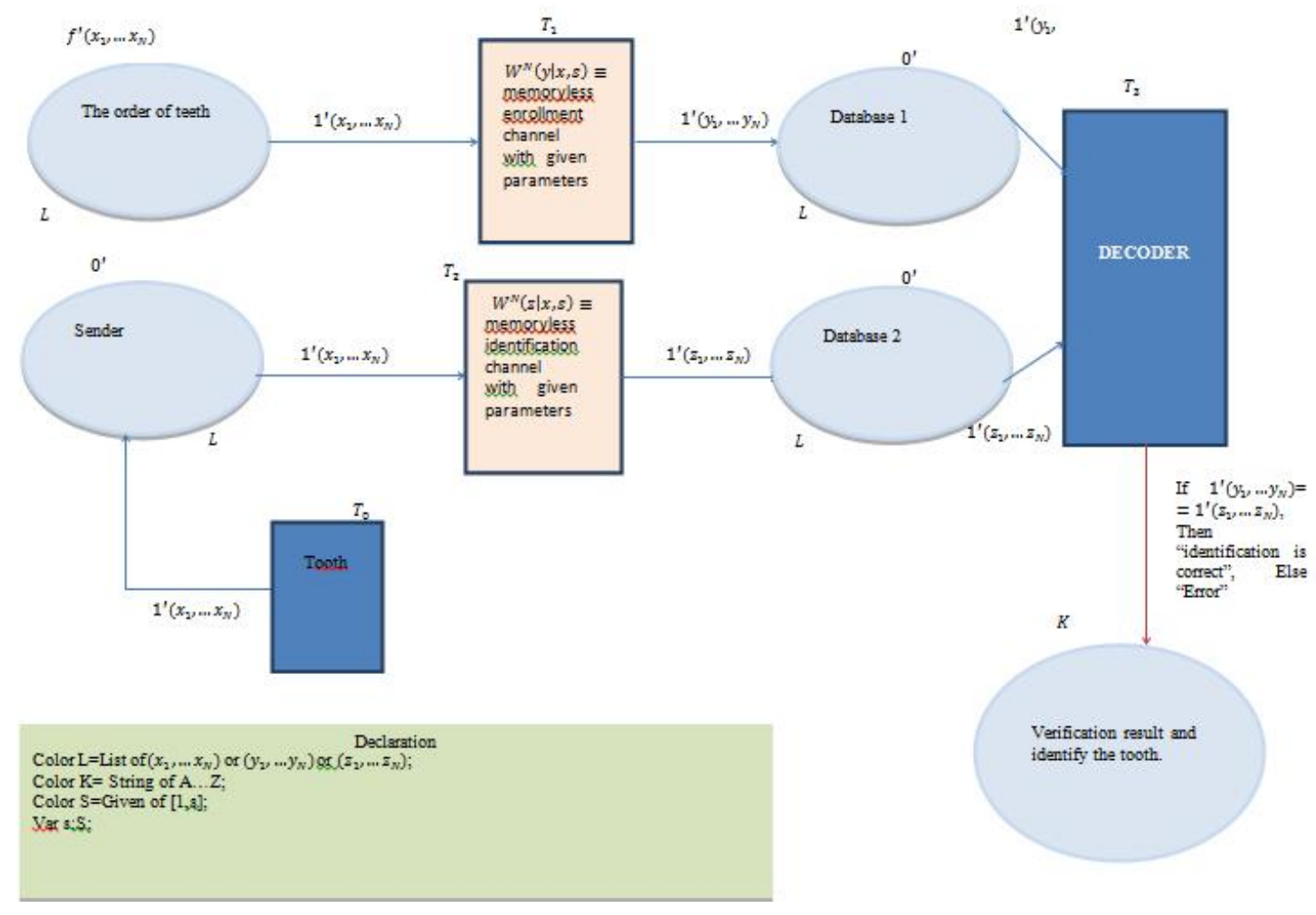

Figure 6. Modelling of Biometric identification system with given parameters using Colored Petri net.

$$
x \in X^{N}, y \in Y^{N}, s \in S^{N}
$$

The resulting identification output sequence $\mathbf{z}$ is compared to the sequences $x(f), f=\{1,2, \ldots, F\}$.

$$
\begin{aligned}
& W^{*}(y \mid x)=\sum_{s} Q(s) W(y \mid x, s), \\
& W^{*}(z \mid x)=\sum_{s} Q(s) W(z \mid x, s)
\end{aligned}
$$

And

$$
\begin{gathered}
P^{*}=\left\{P^{*}(y)=\sum_{x} W^{*}(y \mid x) P(x)\right\} \\
x \in X^{N}, y \in Y^{N} \\
\sum W^{*}(y \mid x) W^{*}(z \mid x) P(x)
\end{gathered}
$$

$W^{*}(z \mid x)=\frac{\sum_{x}}{P^{*}(y)}$.

The channel $W_{s}: Y \rightarrow Z$ is memoryless:

$$
\begin{gathered}
W^{N}(z \mid y)=\prod_{n=1}^{N} W\left(z_{n} \mid y_{n}\right), \\
z \in Z^{N}, y \in Y^{N} .
\end{gathered}
$$

From (1) appointments follows that Biometric Identification Protocol with Given Parameter is brought to Biometric Identification system.
In Fig. 6 Colored Petri Net consists of the following positions: Order of teeth, Sender, Database 1, Database 2, Verification result and the following transitions: $T_{0}, T_{1}, T_{2}, T_{3}$, which have corresponding names. Order of teeth includes $\mathrm{N}$-dimensional biometric data from f-dimensional set. Element $\left(x_{1}, x_{2}, \ldots, x_{n}\right)$ passes through the channel to $T_{1}$ transition, and its corresponding encoded data $\left(y_{1}, y_{2}, \ldots, y_{n}\right)$ is placed in Database 1.

After the firing of $T_{0}$ transition, the element of $\mathrm{f}$ dimensional set is placed in Sender position, after that the result of firing of $T_{2}$ transition, through the respective channel, it is obtained the encoded data $\left(z_{1}, z_{2}, \ldots, z_{n}\right)$, which is placed in the Database 2 position. Through the firing of $T_{3}$ transition, it is checked the correspondence of vectors $\left(y_{1}, y_{2}, \ldots, y_{n}\right)$ and $\left(z_{1}, z_{2}, \ldots, z_{n}\right)$ gets the response: if the identification is correct, or there is an error in the system. The arc, which is directed from $T_{3}$ transition to the Verification result position, is bound by a corresponding logical expression. Then firing of $T_{0}$ transition, the next element can queue up and the cycle can be repeated again.

Order of teeth, Sender, Database 1, Database 2 positions that are attached to the $\mathrm{L}$ type, which is a set of $\mathrm{N}$-dimensional 
vectors. The $\mathrm{K}$ type is attached to Verification result position, which is presented as a type String.

The $S$ type is presented given parameters. The corresponding information about the types is shown in the declaration table.

\section{CONCLUSION}

The Colored Petri net is presented, which simulates the work of the biometric identification protocol with a given parameters. The Colored Petri net allows examining the problems of verification, error detection and functional interactions in biometric identification system. Petri Network, being a graphical representation for modeling system operation, works as high-level programming language and can be used as tool for quality estimation of the modeled system.

In the article, the idea of the Petri net is used to develop the program of the identification system; in addition, it help to describe in detail the algorithm by which one can carefully process 3D models of teeth. The considered properties of Petri nets in fact help to make the proposed software run more quickly, without errors, without deadlocks. The use of Petri nets helps to design optimal models of biometric systems. The results of the research will be used to process biometric data used in medical and technical equipment, which will reduce the time and resource consumption.

\section{REFERENCES}

Ahlswede , R., and Csiszar, 1993. "Common randomness in information theory and cryptography - Part I: Secret sharing", IEEE Trans. Information Theory, vol. IT-39, pp. 1121-1132, July.

Hilson S., 2003. Dental Anthropology. Cambridge University Press.

Jensen, K., 1992. Coloured Petri Nets: Basic Concepts, Analysis Methods and Practical Use. Springer - Verlag, Berlin,

Jensen, K., 1996. Coloured Petri Nets: Basic Concepts, Analysis Methods and Practical Use. Springer,. Vol. 1-3.

Murata, T., 1989. "Petri nets: Properties, Analysis and Applications." Proc. of the IEEE, 77(4),

O'Sullivan, J. A., Schmid, N. A., 2004. "Performance prediction methodology for biometric systems using a large deviations approach", IEEE Trans. On it Signal Proc., vol. 52, no. 10 , pp. 3036-3045.

Pankanti, S., Bolle, R. M. and Jain, A., 2002. "Biometrics The Future of Identification", IEEE Computer, V33, N2, pp. 46-49.
Peterson, J., 1981. Petri Net Theory and the Modelling of Systems.Prentice Hall.ISBN 0-13-661983-5.

Petrosyan, G., Ter-Vardanyan, L., Gaboutchian, A., 2015. "Modeling of Biometric Identification System using the Colored Petri Nets" - The International Archives of the Photogrammetry, Remote Sensing and Spatial Information Sciences, Volume XL-5/W6, 2015 Photogrammetric techniques for video surveillance, biometrics and biomedicine.

Trucel, E., 2006. "Capacity/storage tradeoff in highdimensional identification system", IEEE International Symposium on Information Theory, Washington, USA, pp 1929-1933.

Ullman, J. D., 1998. “Elements of ML Programming,” PrenticeHall, Upper Saddle River.

Willems, F., Kalker, T., Goselig, J., Linnartz, J.-P., 2003. “On the capacity of a biometric identification system", International Symposium on Information Theory, Yokohama, Japan, p. 82,

Zubov A., 2006. Methodological Guidance for Anthropological Analysis of Odontological Materials. ETNO-ONLINE, Moscow. 